\title{
The Importance of Myeloid-Derived Suppressor Cells in the Regulation of Immunosuppressive Mechanisms by Severe Fever with Thrombocytopenia Syndrome
}

\author{
Liping Sun ${ }^{1,2}$, Huixia Zhao², Yajiao $\mathrm{Li}^{3}$, Lizhen $\mathrm{Sun}^{1}$, Huiyu Li ${ }^{3}$, , Shenghua Jie ${ }^{2, ~ * ~}$ \\ ${ }^{1}$ Department of Infectious Diseases, Miyun Teaching Hospital, Capital Medical University, Beijing, PR China \\ ${ }^{2}$ Department of Infectious Diseases, Union Hospital, Tongji Medical College, Huazhong University of Science and Technology, Wuhan, PR \\ China \\ ${ }^{3}$ Center for Stem Cell Research and Application, Union Hospital, Tongji Medical College, Huazhong University of Science and Technology, \\ Wuhan, PR China
}

Email address:

abeycd@126.com (Shenghua Jie), huiyuli26@aliyun.com (Huiyu Li)

${ }^{*}$ Corresponding author

\section{To cite this article:}

Liping Sun, Huixia Zhao, Yajiao Li, Lizhen Sun, Huiyu Li, Shenghua Jie. The Importance of Myeloid-Derived Suppressor Cells in the Regulation of Immunosuppressive Mechanisms by Severe Fever with Thrombocytopenia Syndrome. European Journal of Clinical and Biomedical Sciences. Vol. 4, No. 5, 2018, pp. 63-68. doi: 10.11648/j.ejcbs.20180405.11

Received: November 15, 2018; Accepted: December 13, 2018; Published: January 7, 2019

\begin{abstract}
Objective: Severe fever with thrombocytopenia syndrome (SFTS) is an emerging infectious disease caused by SFTS virus (SFTSV) with a high fatality rate. The objective of our study was to assess the mechanisms of immunofunction through detecting the presence of myeloid-derived suppressor cells (MDSC), granulocyte-colony stimulating factor (G-CFS) and T cells in SFTS patients. Methods: Serum samples from 30 SFTS cases and 20 healthy donors were collected for the test with flow cytometry and sandwich ELISA which contains CD3+, CD4+ T lymphocytes, subsets of MDSC and G-CSF. Results: Granulocytic MDSC (G-MDSC) and monocytic MDSC (M-MDSC) were significantly elevated in SFTS compared to normal control, and G-CSF was expressed at increased frequency. In contrast, CD3+ and CD4+ T lymphocytes were significantly diminished. Further analysis revealed that G-MDSC and G-CSF were higher in severe SFTS infection compared to the patients in mild SFTS infection, and the numbers of $\mathrm{CD} 3+$ and $\mathrm{CD} 4+\mathrm{T}$ lymphocytes showed a more robust pattern of depression. Conclution: In summary, we have characterized an immunosuppressive mechanism in SFTSV infection dependent on G-CSF induction on MDSC and MDSC suppressing T cells.
\end{abstract}

Keywords: Severe Fever with Thrombocytopenia Syndrome (SFTS), Myeloid-derived Suppressor Cells (MDSC), Granulocyte-colony Stimulating Factor (G-CFS), T cells, Immunosuppression

\section{Introduction}

Myeloid-derived suppressor cells (MDSC) are a group of phenotypically heterogeneous myeloid cells that expands during cancer, inflammation and infection, and that plays a remarkable role in suppressing T-cell responses [1]. In humans, MDSC were defined as cells that co-purified with mononuclear cells and granulocyte markers [2]. Though morphologically MDSC consist of a mixture of monocytic and granulocytic cells, MDSC are subdivided into two major groups: granulocytic MDSC (G-MDSC) and monocytic
MDSC (M-MDSC) through identifying specific markers. However, the proportion of G-MDSC and M-MDSC in different tumor models is highly variable and depends on factors that are not yet well understood [3]. Different subsets of MDSC might use different mechanisms to suppress T-cell proliferation. Interestingly, both populations suppressed antigen-specific T-cell proliferation to an equal extent, although by using distinct effector molecules and signal pathways [4]. Features common to all MDSC are their myeloid origin, immature state, biological activity and a remarkable ability to suppress T-cell responses. Many underlying mechanisms of MDSC activity and their specific 
contribution to the pathological processes associated with infectious diseases remain to be elucidated [3].

Recently, it has become clear that the suppressive activity of MDSC requires not only factors that promote their expansion but also induce their activation. And granulocyte-colony stimulating factor (G-CFS) is one of these factors that induce MDSC expansion [5], which is produced mainly by activated $\mathrm{T}$ cells. Although initial analysis and most of the current observations regarding the role of MDSC in immune responses have come from studies in the cancer field, accumulating evidence has shown that MDSC plays an important role in the cellular network involved in the regulation of immune responses in infectious diseases. SFTS is an emerging infectious disease caused by severe fever with thrombocytopenia bunyavirus (SFTSV) with proven impaired $\mathrm{T}$ cell responses and immune exhaustion [6]. However, the precise contribution of SFTSV-induced systemic inflammation to metastasis and the mechanisms regulating systemic inflammation are poorly understood. Our current studies clearly indicate that the expansion of an immunosuppressive MDSC population is frequently observed in the pathological conditions of SFTS. Therefore, it is important to investigate the role of MDSC that is involved in inflammation generation, development and expansion in SFTS patients.

\section{Methods}

\subsection{Patients Enrollment}

A total of 30 adult SFTS patients were prospectively enrolled at Department of Infectious Diseases of Union Hospital, Tongji Medical College, Huazhong University of Science and Technology between May 2014 and September 2015. Ethics Committee approval was obtained from the Research Ethics Committee of Tongji Medical College, Huazhong University of Science and Technology. Clinical data were collected from patients records.

\subsection{Flow Cytometry}

The peripheral blood samples were drawn into ethylene diamine tetraacetic acid (EDTA) anticoagulation tube and processed for the analysis by flow cytometry within $2 \mathrm{~h}$. To determine the frequency and phenotype of MDSC in peripheral blood mononuclear cells (PBMC), flow cytometry was done using the following antibodies: Fluorescein isothiocyanate (FITC)-conjugated anti-CD15, peridinin chlorophyll-protein (PerCP)-labeled anti-CD45 and allophycocyanin (APC)-labeled anti-CD14 and phycoerythrin (PE)-conjugated anti-CD11b. Then to investigate the immunity function, we detected the immune cells in the circulation. The following antibodies (Becton-Dickinson) were used: FITC-conjugated anti-CD3, APC-labeled anti-CD4 and PerCP-labeled anti-CD45. Flow cytometry followed routine procedures. Flow cytometry data were acquired using a FACS Caliber ${ }^{\mathrm{TM}}$ low cytometer (Becton Dickison Crop. the USA.) and analyzed with FlowJo software version 8.8.6 (Tree Star, Ashland, OR).

\subsection{Measurement of $G-C S F$}

The blood plasma was collected from the peripheral blood. The relative amount of G-CSF in blood plasma was determined in a sandwich ELISA using the G-CSF ELISA Kit ( $\mathrm{R} \& \mathrm{D}$ Systems, Abnova, invitrogen) according to standard procedures.

\subsection{Statistical Analysis}

Experimental differences over the controls were analyzed by two-tailed Student's $t$-test. Means \pm standard deviations $(\bar{x} \pm \mathrm{s})$ of at least three experiments are represented. All probability values $P<0.05$ were considered statistically significant. All statistical analyses were performed using the GraphPad Prism 5 (Graph Pad Prism, La Jolla, CA, USA).

\section{Results}

\subsection{Patient Characteristics}

The 30 SFTS patients were admitted from mountainous and urban areas of Henan and Hubei province and they described a history of field and herbosa exposure within two weeks of the illness onset. The mean age was 59 years (range, 40-76 years), and $16(53.3 \%)$ were female. All the patients suffered fever during the disease progression and the mean days were 9.3 days (range, 6-16 days). The mean length of hospital stay was 10.9 days (range, 5-19 days). Five patients of them $(16.7 \%)$ who were 46 to 76 years old had died from severe SFTS with lung infection and heart and respiratory failure in our research. Of these patients, there were 10 patients were severe with an average age of 59 years. Meanwhile, we separated the SFTS patients into mild group and severe group [6-8]. Patient demographics are outlined in Table 1 .

Table 1. Basic characteristics of samples involved in this study.

\begin{tabular}{|c|c|c|c|c|c|c|c|c|c|c|}
\hline \multirow{2}{*}{ group } & \multirow{2}{*}{ number } & \multicolumn{2}{|l|}{$\operatorname{sex}$} & \multicolumn{2}{|l|}{ Age } & \multicolumn{2}{|c|}{ Duration of fever } & \multicolumn{2}{|c|}{ Length of Hospital stay } & \multirow[t]{2}{*}{ Death } \\
\hline & & $\mathbf{F}$ & M & Median & Range & Median & Range & Median & Range & \\
\hline Control & 20 & 16 & 4 & 32 & $17-60$ & - & - & - & - & - \\
\hline SFTS & 30 & 16 & 14 & 59.5 & $40-76$ & 8 & $6-16$ & 9.5 & $5-19$ & 5 \\
\hline Mild & 20 & 12 & 8 & 58.5 & $44-72$ & 8.5 & $6-12$ & 9.5 & $6-13$ & - \\
\hline Severe & 10 & 4 & 6 & 61.5 & $40-76$ & 10.5 & $7-16$ & 12.5 & $5-19$ & 5 \\
\hline
\end{tabular}




\subsection{MDSC Increase in SFTS Patients}

In an attempt to determine the regulatory function of MDSC in SFTS patients, we compared the amount of peripherally circulating MDSC in 30 SFTS patients compared with control healthy volunteers, and determined their inhibitory properties. Our results demonstrate that both M-MDSC and G-MDSC increase in SFTS patients and are significant $(p<0.05)$. However, severe patients had a significant increase in G-MDSC expressing compared to the mild patients $(p<0.05)$, while there were no obvious statistical significance for M-MDSC $(p>0.05)$ in Figure 1.

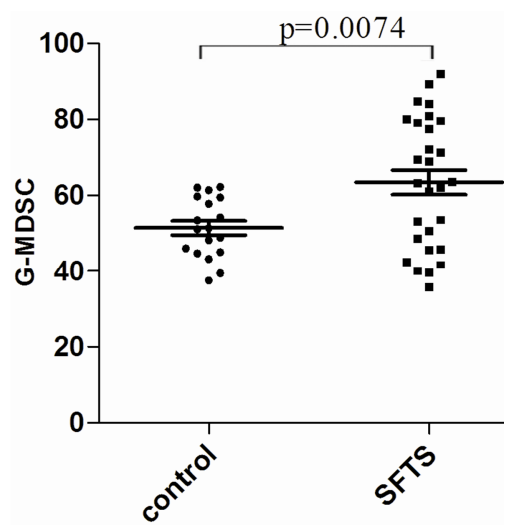

A

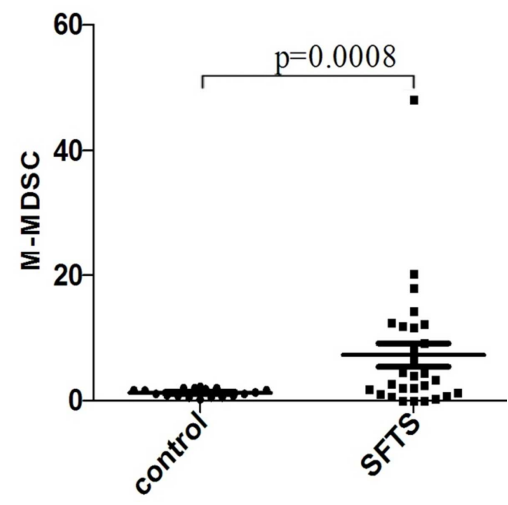

B

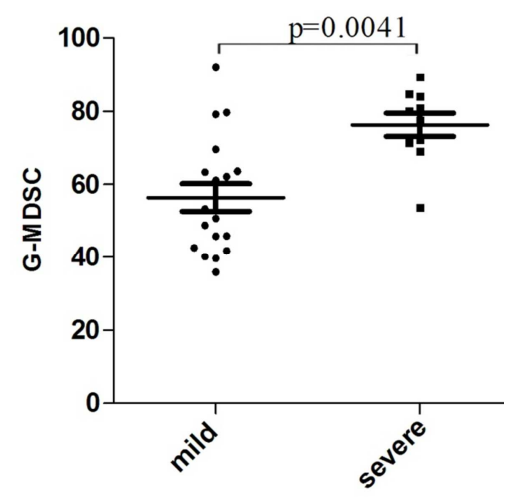

C

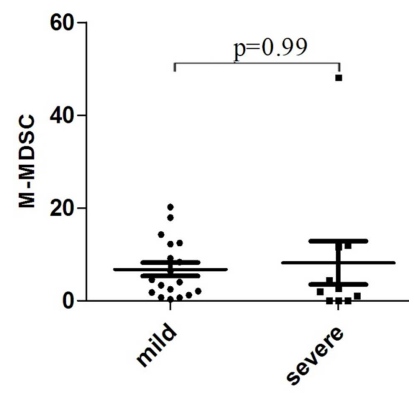

D

Figure 1. Comparison of the levels of G-MDSC and M-MDSC, as determined by flow cytometry. (A) The levels of G-MDSC in 30 SFTS patients and 20 healthy donors. (B) The levels of M-MDSC were compared between SFTS patients and healthy donors. (C) The levels of G-MDSC were compared between 10 severe cases and 20 mild cases. (D) The levels of M-MDSC were in severe cases vs mild cases. Data were expressed as Means with SEM.

\subsection{G-CSF Increase in SFTS Patients}

The notion of MDSC has led to a comprehensive understanding of immune suppression of SFTS patients. To test the hypothesis that MDSC production is causally linked to G-CSF generation, the levels of G-CSF in the SFTS patients and normal control were detected respectively. G-CSF was expressed at increased frequency in SFTS patients, with the highest level of expression frequently seen in severe SFTS patients, and the differences were significant $(\mathrm{p}<0.05)$ in Figure 2. Not surprisingly, G-CSF levels increased with increasing patients' condition. The studies extend observations that it is correlated between G-CSF and MDSC in SFTS patients.

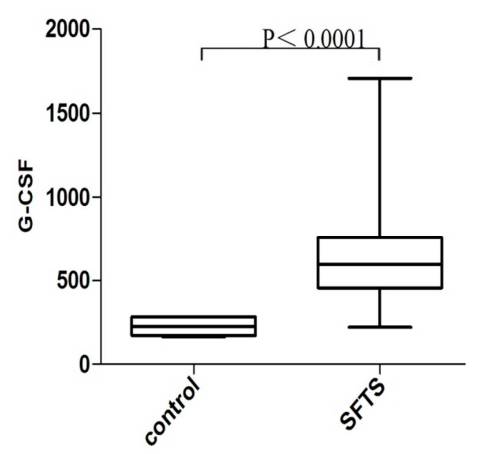

A

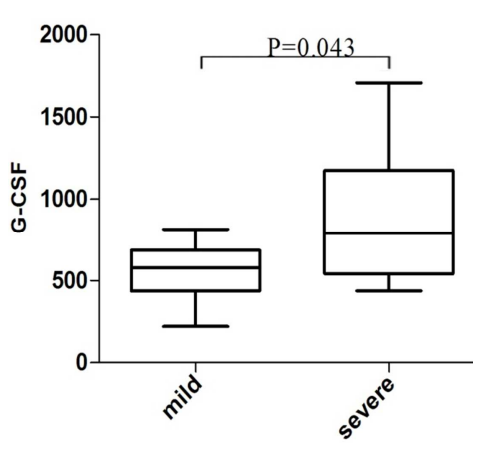

B

Figure 2. Comparison of the levels of G-CSF. (A) The levels of G-CSF in 30 SFTS patients and 20 healthy donors. (B) The levels of G-CSF were compared in 20 mild cases vs 10 severe cases. 


\subsection{Immunofunction in SFTS Patients}

To determine whether differences in MDSC levels affected immunofunction, the lymphocyte subsets were analyzed in SFTS patients as before [6]. The major differences in CD3+ and CD4+ were observed between the control normal and SFTS patients. The CD3+ and CD4+ T lymphocytes were decreased significantly in SFTS with the $p<0.01$. Levels in patients with mild and severe SFTSV infection were compared. In severe group, the percentages of CD3+ and CD4+ were significantly lower than that in mild group obviously $(\mathrm{p}<0.05)$ in Figure 3.

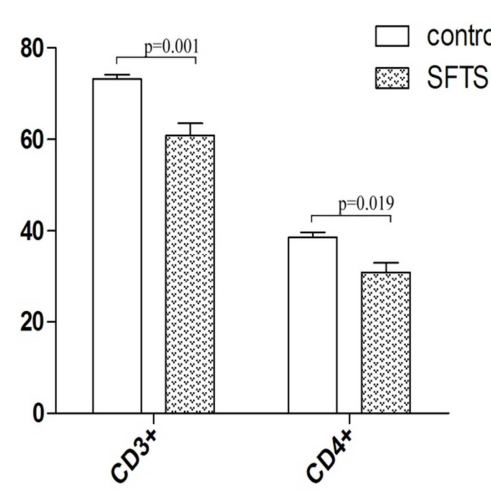

A

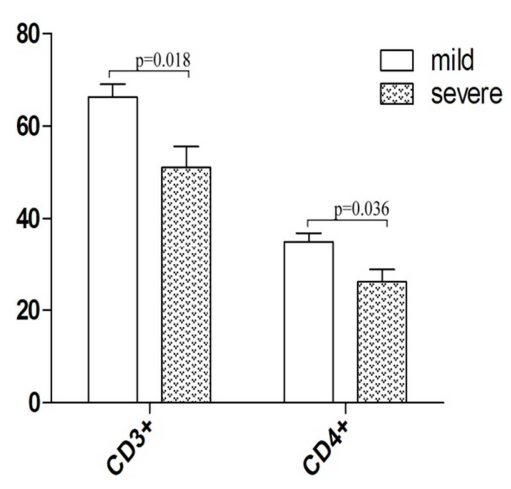

B

Figure 3. Comparison of percentage of $\mathrm{CD} 3+, \mathrm{CD} 4+$ T lymphocyte. (A) The percentage of lymphocytes in 30 SFTS patients and 20 healthy donors. (B) The percentage of lymphocytes were compared between 10 severe cases and 20 mild cases.

\section{Discussion}

In recent years we have witnessed high interest for SFTS, so the pathogenesy has become evident gradually. In this article we focused on the mechanism of immune suppression and provided opportunities to better understand the immune responses for SFTS patients. Our results show that a correlation exists between the presence of G-CFS, MDSC and $\mathrm{T}$ cells.

The frequency of MDSC population increased further significantly comparing with the normal control in SFTS patients. A significantly higher percentage of G-MDSC was seen in severe SFTS group, and there was no significant difference in M-MDSC population comparing with the mild group. Differential expression analysis of the data revealed that the expanded population of MDSC in the severe SFTS patients resembles persistent infection induced MDSC. MDSC were recently identified as a major factor involved in immune suppression associated with infectious diseases and have the ability to suppress $\mathrm{T}$ cell proliferation [4]. The previous data also present a possibility to resolve the results that $\mathrm{CD} 3+$ and $\mathrm{CD} 4+$ were proposed to be major targets of inflammation-mediated $\mathrm{T}$ cell inhibition [6]. Therefore, the MDSC suppress the proliferation of $\mathrm{T}$ cells and it was capable of inhibiting CD3+ and CD4+ T cell proliferation. MDSC mainly reside in spleens, bone marrow and peripheral blood of healthy individuals and expand upon chronic stimulation of the immune system, revealing suppressive function mostly toward repeatedly stimulated immune effector cells [9, 10]. Current thinking suggests that MDSC acquire suppressive function only after exposure to factors present in inflammatory microenvironments [11]. Accordingly, we presumed that MDSC suppressed the proliferation of stimulated rather than common T cells.

In prospective study, the sera G-CSF levels were significantly higher in SFTS patents compared to the control. High levels of G-CSF were observed in severe SFTS patients, whereas nominal amounts were detectable in the mild group. G-CSF levels in SFTS patients paralleled the high levels of G-MDSCs found in the same group. Waight et al had demonstrated that G-CSF levels had a perfect effect on G-MDSC subset, and remained largely unaffected for M-MDSC [12]. This was in perfect agreement with our results. However, it remained to be determined whether the effect of G-CSF on patient's condition acted through a MDSC-induced mechanism. Through comparing the levels of G-CSF to the levels of MDSC generated in SFTS patients, we had gained the insights into the role of G-CSF as a potential mediator of G-MDSC development. Our study demonstrated that inflammation-derived G-CSF facilitates MDSC generation which displays both immunosuppressive and proinflammatory activities.

We investigated the role of G-CSF in the development of MDSC, and demonstrated that the presence of MDSC correlated significantly with elevated number of G-CSF in circulation. The high level of G-CSF expression in SFTS patients could well assist MDSC recruitment. A report had shown that the combination of G-CSF and IL-6 allowed for a rapid generation of MDSC from precursors present in human bone marrow [13]. The data indicated that G-CSF is produced at high levels in severe patients, and represents a potentially critical player for altered myelopoiesis and granulocytic MDSC generation. The patients with severe neutropenia are prone to bacterial infection for lacking G-CSF, because G-CSF plays a critical role during neutrophil development and in emergency granulopoiesis. Routinely G-CSF is administered to the patients with neutropenia to boost the number of neutrophils. Nevertheless, exogenous G-CSF can inhibit innate immune responses through the recruitment of MDSCs [14]. And another study has shown 
that administration of G-CSF to mobilize stem cells is a companied by an expansion of G-MDSC [15]. It had been demonstrated a direct correlation between the level of G-CSF and the number of G-MDSC that abrogating G-CSF production using RNAi resulted in a reduced accumulation of G-MDSC [12]. These studies suggest that high levels of G-CSF can hamper the innate immune response by promoting the expansion of MDSC in SFTS patients.

While MDSC have been described to positively regulate cell-mediated immune responses by inhibiting $\mathrm{T}$ cell proliferation and promoting regulatory $\mathrm{T}$ cell expansion in cancer patients [16], the presence and immune regulatory function also plays important roles in SFTS patients. MDSC play an important role during benign inflammatory conditions in vivo [17, 18], and regulate cell mediated immune response in vivo during inflammatory responses [19]. G-MDSC in humans that inhibit $\mathrm{T}$ cell proliferation via an ARG1-mediated mechanism are simply activated granulocytes [20]. Therefore, inflammatory factors and/or activated $\mathrm{T}$ cells are necessary for activation of suppressive function in MDSC. In our prospective study, the striking findings were the significant depression in peripheral blood CD3+ and CD4+ in patients with SFTSV infection. The interaction of MDSC with $\mathrm{T}$ cells has been studied extensively. In order to modulate the function and proliferation of $\mathrm{T}$ cells, neutrophils or G-MDSC need to come in contact with or in close proximity to T cells [19]. A study also showed that, in patients with gastric cancer, G-MDSC isolated from the tumor site suppressed $\mathrm{T}$ cells in a contact-dependent manner [21]. It also shows that $\mathrm{T}$ cells can proliferate at sites of viral infection, which is exactly the place where vast amounts of neutrophils are found [22]. Thus, MDSC can inhibit cellular immunofunction in SFTS patients, this could be a mechanism through which overexpression of MDSC can suppress $\mathrm{T}$ cell function. The expansion and activation of MDSC efficiently suppress the proliferation of freshly activated CD3+ and CD4+ effector cells in SFTS patients. Therefore, acute SFTSV infection, which induces T-cell activation and increased production of G-CSF, also leads to the expansion of MDSC.

\section{Conclusion}

Taken together, the results suggest mechanisms to account for immunosuppression in SFTSV infections. Thus, SFTSV-derived inflammatory G-CSF facilitate to produce large amounts of MDSC that in turn suppress the function of $\mathrm{T}$ cells. This immunosuppression is thought to facilitate the dissemination of SFTSV in the infected host. It was presumed that SFTSV maximizes its chance of metastasizing by evoking a systemic inflammatory cascade in SFTS patients.

\section{Acknowledgements}

This work was supported by the National Nature Science Foundation of China (grant: 81171638). We would like to thank the teachers at the Center for Stem Cell Research and Application for technical assistance and the professors in the Department of Infection Disease for their help in collecting samples.

\section{Conflict of Interest}

All the authors do not have any possible conflicts of interest.

\section{Ethical Approval}

Ethics Committee approval was obtained from the Research Ethics Committee of Tongji Medical College, Huazhong University of Science and Technology.

\section{References}

[1] Nagaraj S, Schrum AG, Cho HI, Celis E, Gabrilovich DI Mechanism of $\mathrm{T}$ cell tolerance induced by myeloid-derived suppressor cells. J Immunol. 2010, 184: 3106-3116.

[2] Zea AH, Rodriguez PC, Atkins MB, Hernandez C, Signoretti $\mathrm{S}$, Zabaleta J, McDermott D, et al. Arginase-producing myeloid suppressor cells in renal cell carcinoma patients: a mechanism of tumor evasion. Cancer Res. 2005, 65: 3044-3048.

[3] Youn JI, Gabrilovich DI. The biology of myeloid-derived suppressor cells: the blessing and the curse of morphological and functional heterogeneity. Eur J Immunol. 2010, 40: 2969-2975.

[4] Gabrilovich DI, Nagaraj S. Myeloid-derived suppressor cells as regulators of the immune system. Nat Rev Immunol. 2009, 9: $162-174$

[5] Serafini P, Carbley R, Noonan K, Tan G, Bronte V, et al. High-dose GM-CSF-producing vaccines impair the immune response through the recruitment of myeloid suppressor cells. Cancer Res. 2004, 64: 6337-6343.

[6] Sun LP, Hu YJ, Niyonsaba A, Tong QX, Li HY, Jie SH. Detection and evaluation of immunofunction of patients with Severe Fever with Thrombocytopenia Syndrome. Clin Exp Med. 2014, 14: 389-395.

[7] Jie SH, Zhou Y, Sun LP, Liang KW, Yi XL, Li HY. Close correlation between development of MODS during the initial $72 \mathrm{~h}$ of hospitalization and hospital mortality in severe fever with thrombocytopenia syndrome. J Huazhong Univ Sci Technol. 2013, 33: 81-85.

[8] Sun LP, Li HY, Yi XL, Niyonsaba A, Wang NF, Jie SH. Detection and clinical significance of circulating microvesicles in severe fever with thrombocytopenia syndrome. J infection international. 2013, 2: 49-54.

[9] Baniyash M. TCR ל-chain downregulation: curtailing an excessive inflammatory immune response. Nature Reviews Immunology. 2004, 4: 675-687.

[10] Serafini P, De Santo C, Marigo I, Cingarlini S, Dolcetti L, et al.. Derangement of immune responses by myeloid suppressor cells. Cancer Immunol Immunother. 2004, 53: 64-72. 
[11] Greifenberg V, Ribechini E, Rößner S, Lutz MB. Myeloid-derived suppressor cell activation by combined LPS and IFN-gamma treatment impairs DC development. Eur J Immunol.2009, 39:2865-2876.

[12] Waight JD, Hu Q, Miller A, Liu S, Abrams SI. Tumor-Derived G-CSF Facilitates Neoplastic Growth through a Granulocytic Myeloid-Derived suppressor cell-dependent mechanism. PLoS One. 2011, 6: e27690.

[13] Marigo I, Bosio E, Solito S, Mesa C, Fernandez A, Dolcetti L, Ugel $\mathrm{S}$, et al. Tumor-induced tolerance and immune suppression depend on the $\mathrm{C} / \mathrm{EBP}$ beta transcription factor. Immunity. 2010, 32: 790-802.

[14] Trikha P, Carson WE $3^{\text {rd }}$. Signaling pathways involved in MDSC regulation. Biochim Biophys Acta, 2014, 1846: 55-65.

[15] Luyckx A, Schouppe E, Rutgeerts O, Lenaerts C, Fevery S, et al.. G-CSF stem cell mobilization in human donors induces polymorphonuclear and mononuclear myeloid-derived suppressor cells. Clin Immunol. 2012, 143: 83-87.

[16] Gabrilovich DI, Ostrand-Rosenberg S, Bronte V. Coordinated regulation of myeloid cells by tumours. Nat Rev Immunol.2012, 12: 253-268.

[17] Bronte V, Apolloni E, Cabrelle A, Ronca R, Serafini P, Zamboni P, Restifo NP, Zanovello P. Identification of a CD11b $(+) / \mathrm{Gr}-1(+) / \mathrm{CD} 31(+)$ myeloid progenitor capable of activating or suppressing CD8(+) T cells. Blood. 2000, 96: 3838-3846.

[18] Marhaba R, Vitacolonna M, Hildebrand D, Baniyash M, Freyschmidt-Paul P, Zöller M. The Importance of Myeloid-Derived Suppressor Cells in the Regulation of Autoimmune Effector Cells by a Chronic Contact Eczema. J Immunol. 2007, 179: 5071-5081.

[19] Haverkamp JM, Crist SA, Elzey BD, Cimen C, Ratliff TL. In vivo suppressive function of myeloid-derived suppressor cells is limited to the inflammatory site. Eur J Immunol. 2011, 41: 749-759.

[20] Rodriguez PC, Ernstoff MS, Hernandez C, Atkins M, Zabaleta $\mathrm{J}$, et al.. Arginase I-producing myeloid-derived suppressor cells in renal cell carcinoma are a subpopulation of activated granulocytes. Cancer Res. 2009, 69: 1553-1560.

[21] Zhuang Y, Peng LS, Zhao YL, Shi Y, Mao XH, et al.. CD8(+) $\mathrm{T}$ cells that produce interleukin-17 regulate myeloid-derived suppressor cells and are associated with survival time of patients with gastric cancer. Gastroenterology. 2012, 143: 951-962.

[22] Heidema J, Lukens MV, van Maren WW, van Dijk ME, Otten $\mathrm{HG}$, et al. CD8 $+\mathrm{T}$ cell responses in bronchoalveolar lavage fluid and peripheral blood mononuclear cells of infants with severe primary respiratory syncytial virus infections. J Immunol. 2007, 179:8410-8417. 